\title{
Aspectos Metodológicos Relacionados aos Sistemas Manométricos Utilizados em Estudos Hemodinâmicos
}

\author{
Edson Antonio Bregagnollo', Fábio Cardoso Carvalho', \\ Isamara Fernanda Bregagnollo ${ }^{2}$, Jorge Sadame Hirata ${ }^{1}$
}

\section{RESUMO}

Atualmente, diante das técnicas atuais, a manometria tem sido relegada a plano secundário durante a cateterização cardíaca. No entanto, ainda fornece importantes informações para identificação e avaliação das doenças cardiovasculares. Os dados coletados durante os exames possibilitam a obtenção de variáveis quantitativas e qualitativas, as quais podem ser comparadas aos padrões normais. Os sistemas manométricos são compostos por transdutor, amplificador e registrador, que, em conjunto, devem espelhar com fidelidade a morfologia e os valores das variáveis analisadas. Para atingir esse objetivo, é necessário desempenho adequado de todos os componentes. Se uma determinada informação é de extrema relevância, o operador deve gastar tempo suficiente para obtê-la de maneira inequívoca. Assim, o operador deve estar familiarizado com os sistemas manométricos e com as fontes de erro relacionadas com as técnicas de registro, cateteres, conectores e fluidos. Com os fundamentos analisados neste manuscrito, salientamos que deve ser dispensada atenção às ondas de pressão usadas nas interpretações da fisiopatologia das doenças cardiovasculares.

DESCRITORES: Pressão arterial. Manometria. Determinação da pressão arterial, instrumentação.

\section{SUMMARY}

Methodologics Aspects Related to Manometric System Used in Hemodynamics Studies

Cardiac catheterization and ancillary procedures provide invaluable information for the detection of heart disease and the assessment of cardiac function. Raw data are collected in the course of the investigation and then processed, yielding hemodynamic and mechanical variables which can be evaluated qualitatively and quantitatively against the known standards. Precise recordings are clearly indispensable for a reliable hemodynamic evaluation: to accomplish this objective, the use of an appropriate instrument system must be made. A modern instrument system for physiologic recording consists of three main components, namely, transducer, amplifier, and indicator. The adequacy of an instrument system depends on the performance of its components: stability, sensitivity, linearity and an adequate frequency response. If the information is considered important enough to perform hemodynamic measurements, the operators should take the time to obtain pressure waveforms that are reliable and unequivocal, separating artifact from pathology. With the fundamentals analyzed in this paper, we would like to point out that, all due attention should be paid to the pressure waves used in the interpretation of the physiopathology of cardiovascular illness.

DESCRIPTORS: Blood pressure. Manometry. Blood pressure determination, instrumentation.
A pressão sanguínea é a força gerada pela atividade mecânica do coração, transmitida por um meio fluido, o sangue, em forma de ondas, com características distintas, dependendo da localização no sistema cardiovascular ${ }^{1,2}$. Do ponto de vista físico,

\footnotetext{
1 Setor de Hemodinâmica do Hospital das Clínicas da Faculdade de Medicina de Botucatu - UNESP, Botucatu, SP.

2 Faculdade de Medicina de Marília - FAMEMA, Marília, SP. Correspondência: Edson Antonio Bregagnollo. Rua Capitão Andrade, 567 - Bairro Alto - Botucatu, SP - CEP 16801-545 Fone/Fax: (14)3811-6121 • E-mail: eantoniobr@gmail.com Recebido em: 7/4/2007 • Aceito em: 6/9/2007
}

pressão é definida como a força exercida por unidade de área. No sistema CGS, a unidade de medida da pressão é dynas $/ \mathrm{cm}^{2}$ (bar). No Sistema Internacional de Medidas, a unidade de pressão é o Pascal $(\mathrm{Pa})$, que é igual a $1 \mathrm{Newton} / \mathrm{m}^{2}$. Na maioria dos laboratórios de Hemodinâmica, os valores das pressões são expressos em milímetros de mercúrio $(\mathrm{mmHg})$, correspondendo à altura de uma coluna líquida cujo peso exerce determinada força na unidade de área de aplicação (membrana do transdutor de pressão) ${ }^{3}$. Um mmHg é definido como 1/760 da pressão exercida por uma coluna de mercúrio de $760 \mathrm{~mm}$ de altura, considerando-se o nível do mar, a aceleração da gravidade de $980,77 \mathrm{~cm} / \mathrm{s}^{2} \mathrm{e}$ a densidade do mercúrio de 13,59 $\mathrm{g} / \mathrm{cm}^{3}$. Em alguns 
Bregagnollo EA, et al. Aspectos Metodológicos Relacionados aos Sistemas Manométricos Utilizados em Estudos Hemodinâmicos. Rev Bras Cardiol Invas 2007; 15(4): 421-431.

laboratórios, são, eventualmente, utilizadas outras unidades de medidas, tais como o Pascal e o quilopascal $(\mathrm{kPa})$. As equivalências entre algumas unidades de medidas de pressão, mais comumente utilizadas na prática diária (clínica e experimental), estão relacionadas na Tabela 1. Para se converter centímetros de solução fisiológica em $\mathrm{mmHg}$, multiplica-se pelo fator 0,76.

Quando um fluido, tal como o sangue, se encontra em movimento, a pressão é uma medida da energia disponível para o fluxo. De acordo com o teorema de Bernoulli, em uma corrente líquida, em que não há perda de energia (calor, atrito, etc.), a somatória de todas as formas de energia é constante. Nessas condições, a energia total do sangue em movimento é representada por: pressão absoluta + energia cinética + energia potencial gravitacional (hidrostática determinada pela posição relativa que se admite como nível de referência zero $)^{3}$. Assim, quando o cateter se encontra no interior do sistema circulatório, os valores da pressão são distintos caso o orifício distal se encontre no mesmo sentido ou em sentido contrário à corrente sanguínea. No primeiro caso, a pressão absoluta (exercida perpendicularmente à parede do vaso ou à cavidade cardíaca) soma-se à energia cinética, enquanto no segundo a pressão absoluta se reduz em função da energia cinética. Em condições de repouso, as diferenças são pequenas e não comprometem, de modo significativo, a interpretação dos resultados obtidos na rotina diária. Por exemplo, em repouso, a energia cinética na aorta humana tem o valor aproximado de 3,75 $\mathrm{mmHg}$. Por outro lado, quando nos deparamos com situações que fogem aos padrões normais vigentes nas condições de repouso (esforço físico), os valores da energia cinética podem atingir cifras de até $94 \mathrm{mmHg}$, prejudicando completamente a análise dos dados obtidos ${ }^{1-3}$. Quando as pressões são utilizadas para determinação de alguns índices de contratilidade e relaxamento miocárdico, tais como a elastância sistólica final (ESF) e a primeira derivada temporal da pressão ventricular esquerda $(\mathrm{dP} / \mathrm{dt})$, o ideal é o emprego de cateter manômetro; na impossibilidade deste, as características dinâmicas dos sistemas manométricos precisam ser minuciosamente conhecidas, a fim de não se incorrer em erros graves ${ }^{4-8}$.

\section{PRESSÃO ABSOLUTA E PRESSÃO RELATIVA}

A pressão que uma coluna líquida exerce sobre uma superfície é igual àquela exercida pelo líquido mais a pressão atmosférica local (pressão absoluta). A pressão atmosférica varia de acordo com a altitude em relação ao nível do mar e também com as condições climáticas (umidade relativa e temperatura). Por exemplo, ao nível do mar, a pressão atmosférica é de $760 \mathrm{mmHg}$, enquanto a 2.500 metros de altitude seu valor é de, aproximadamente, $567 \mathrm{mmHg}$. Assim, trabalhar com valores absolutos de pressão é complexo, visto que cada laboratório deveria ter seus próprios padrões de normalidade e os mesmos adequadamente corrigidos

\section{TABELA 1 \\ Equivalências de medidas utilizadas em Hemodinâmica}

\begin{tabular}{ll}
\hline $1 \mathrm{mmHg}$ & $1.333,23 \mathrm{dyn} / \mathrm{cm}^{2}$ \\
$1 \mathrm{mmHg}$ & $133,30 \mathrm{~Pa}$ \\
$1 \mathrm{mmHg}$ & $0,1333 \mathrm{kPa}$ \\
$1 \mathrm{mmHg}$ & $13,1 \mathrm{~cm}$ de solução fisiológica \\
$1 \mathrm{mmHg}$ & $12,8 \mathrm{~cm}$ de sangue
\end{tabular}

$\mathrm{mmHg}=$ milímetros de mercúrio; dyn $/ \mathrm{cm}^{2}=$ dinas/centímetros quadrados; $\mathrm{Pa}=$ Pascal; $\mathrm{kPa}=$ quilopascal; $\mathrm{cm}=$ centí metros.

para as variações climáticas no momento da cateterização cardíaca. Outro importante inconveniente seria a dificuldade de se comparar dados obtidos em diferentes laboratórios ou no mesmo laboratório em épocas distintas. No entanto, na prática diária, esse tipo de ajuste, provavelmente, teria mínimo efeito real. Habitualmente, no laboratório de Hemodinâmica, as pressões são registradas com o auxílio de um manômetro externo (transdutor), que fornece sempre a diferença entre a pressão sanguínea intracavitária e a pressão atmosférica (pressão relativa). Isso é feito colocandose uma das vias, a câmara do transdutor, já preenchida com soro, em contato com a atmosfera, mantendose a outra fechada. Nessa condição, admite-se, convencionalmente, valor zero para a pressão atmosférica. Esse procedimento permite que, em todos os laboratórios de Hemodinâmica, as pressões registradas sejam normalizadas para as condições atmosféricas locais.

\section{PLANO DE REFERÊNCIA ZERO}

O conjunto cateter + transdutor de pressão + vasos e cavidades cardíacas forma um sistema de vasos comunicantes $^{3,9}$ (Figura 1A). Nesse sistema, podem ser estabelecidas três situações: 1 . quando o transdutor se encontra no mesmo plano da ponta do cateter, os valores das pressões, em uma determinada cavidade, serão iguais em qualquer ponto que se encontre no mesmo plano horizontal; 2. quando o transdutor ocupa um plano inferior àquele da ponta do cateter, ao valor relativo da pressão soma-se a altura da coluna de líquido compreendida entre os planos horizontais, que contém a ponta do cateter e o transdutor (h'); 3. quando o transdutor se posiciona acima do plano da ponta do cateter, a pressão registrada se reduz (h). Cada laboratório de Hemodinâmica deve, portanto, padronizar o plano de referência zero a ser usado na rotina diária.

A determinação adequada do plano de referência zero é um pré-requisito importante para a determinação exata das pressões intracardíacas ${ }^{10,11}$. Na prática diária, são utilizados diferentes níveis de referência zero: 1. manúbrio esternal; $2.5 \mathrm{~cm}$ abaixo do manúbrio; 3 . $10 \mathrm{~cm}$ acima do tampo da mesa; 4. linha axilar média; 
Bregagnollo EA, et al. Aspectos Metodológicos Relacionados aos Sistemas Manométricos Utilizados em Estudos Hemodinâmicos. Rev Bras Cardiol Invas 2007; 15(4): 421-431.

5. metade da espessura torácica; e 6. eixo flebostático. O plano mais usado é a linha axilar média, visto que corresponde, aproximadamente, ao plano da conexão das veias cavas no átrio direito, sendo denominado eixo flebostático (definido como a linha que une o plano frontal do paciente com o plano que inclui a linha axilar média e o plano horizontal, que passa pela união da quarta cartilagem costal com o externo). Quando o eixo flebostático é usado como nível de referência zero, admite-se ser possível comparar as pressões das diversas cavidades cardíacas, mesmo que o paciente não se encontre em decúbito dorsal horizontal. Chandraratna ${ }^{9}$ comparou o valor da distância média entre a parede anterior do tórax e o meio do átrio esquerdo determinado com ecocardiografia, tomado como plano de referência zero em pacientes com e sem cardiopatia. Neste estudo, foi evidenciado que o nível $5 \mathrm{~cm}$ abaixo do manúbrio implica, sistematicamente, erro, enquanto o nível $10 \mathrm{~cm}$ acima do tampo da mesa e metade da espessura torácica não apresentaram diferenças significativas em relação àqueles em que o meio do átrio esquerdo foi determinado com auxílio de ecocardiografia e tomado como plano de referência zero. Considerando-se que a pressão do átrio esquerdo reflete a pressão de enchimento do ventrículo esquerdo, importante fator determinante do desempenho cardíaco, a posição do transdutor em relação ao paciente assegura obtenção de valores pressóricos corretos e seus parâmetros derivados (índices de contratilidade e relaxamento miocárdico, estresse parietal, trabalho cardíaco, resistências vasculares e outros que utilizam os valores pressóricos para cálculo). Essas recomendações são também importantes durante avaliações hemodinâmicas à beira do leito, quando se toma a pressão "capilar pulmonar encunhada" como estimativa da pressão diastólica final do ventrículo esquerdo². Não é demasiado lembrar que a pressão "capilar pulmonar encunhada" só deve ser tomada como estimativa da pressão de enchimento do ventrículo esquerdo na ausência de doença pulmonar obstrutiva crônica, estenose mitral ou doenças oclusivas das veias pulmonares.

\section{CARACTERÍSTICAS FÍSICAS DOS SISTEMAS MANOMÉTRICOS}

Os sistemas manométricos são constituídos por três componentes: transdutor de pressão, amplificador e indicador ${ }^{2,11}$.

O transdutor é o dispositivo eletromecânico que transforma a energia mecânica (pressão) em sinal elétrico, de fácil medida, que varia em função direta com a magnitude das oscilações pressóricas. São exemplos de dispositivos eletromecânicos aqueles compostos de massa-mola-amortecedor (Figura 1B). A massa é o elemento inercial (fluido que preenche o sistema); a mola, o elemento elástico (membrana do transdutor, características elásticas dos cateteres e extensores); e o amortecedor, a forma de dissipar energia do sistema (atrito, viscosidade do fluido, calor). Quando a massa é deslocada de sua posição de equilíbrio e subitamente liberada, o sistema se movimenta em uma série de oscilações com determinada freqüência, cujas ondas apresentam amplitudes decrescentes em torno do ponto de equilíbrio. A freqüência de oscilações, expressa em ciclos por segundo (cps), denomina-se freqüência natural de ressonância do sistema manométrico ${ }^{2,3,11}$. Quanto menor a massa oscilante (volume do líquido no interior do cateter e transdutor), quanto maior a rigidez do elemento elástico e quanto menor o grau de amortecimento mais elevada será a freqüência natural de ressonância. Quando uma variação súbita de pressão é aplicada no sistema manométrico, três tipos de respostas podem ser obtidos (Figura 2A): 1. o sinal de saída atinge rapidamente o valor de entrada, supera-o e é seguido por uma série de oscilações na freqüência

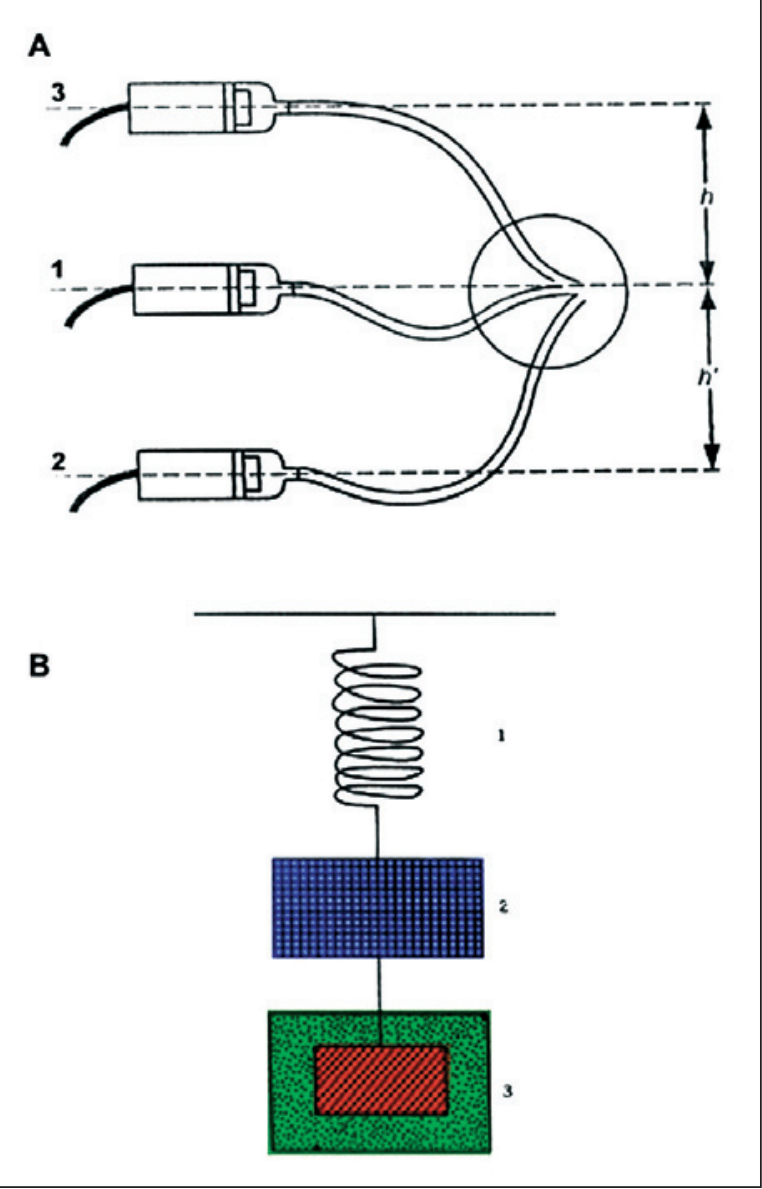

Figura 1 - A- O transdutor, o cateter e a cavidade cardíaca onde se encontra a extremidade distal do mesmo formam um sistema de vasos comunicantes: 1. plano de referência zero; 2 . ocorrência de condição inversa quando se desloca o transdutor a uma altura h' abaixo do plano de referência zero; 3 . se o transdutor é elevado a uma altura $\mathrm{h}$ da pressão registrada, deve-se subtrair a pressão exercida pela coluna líquida h. B- Esquema dos componentes físicos de um transdutor de pressão (Stathan): 1. mola; 2. massa; 3. amortecedor. 
Bregagnollo EA, et al. Aspectos Metodológicos Relacionados aos Sistemas Manométricos Utilizados em Estudos Hemodinâmicos. Rev Bras Cardiol Invas 2007; 15(4): 421-431.

natural de ressonância e amplitudes progressivamente menores, até se estabilizarem no valor do sinal de entrada (nessa condição, trata-se de um sistema com baixo grau de amortecimento, no qual prevalecem os fatores inerciais, favorecendo o aparecimento de artefatos nas curvas de pressão em decorrência da ressonância); 2. o sinal de saída atinge lentamente e sem oscilações o valor do sinal de entrada (nesse caso, predomina o amortecimento e as curvas pressóricas irão apresentar velocidade de aumento e de decréscimo reduzida - traçado amortecido); e 3. o valor do sinal de saída atinge rapidamente e supera ligeiramente o sinal de entrada e logo se estabiliza (nesse sistema, há equilíbrio entre as forças inerciais e amortecimento). Em resumo, os três sistemas diferem basicamente quanto ao grau de amortecimento, sendo muito baixo no primeiro, crítico no segundo e intermediário no terceiro. O último sistema é mais apropriado para registros pressóricos nos laboratórios de Hemodinâmica.

O amplificador aumenta o sinal elétrico de saída a um valor que possibilita que seja percebido e medido. Os dispositivos atuais funcionam com uma onda sinusoidal portadora, que, após ser retificada e filtrada, constitui a curva de pressão que é enviada ao indicador (Figura 2B).

O indicador executa a representação gráfica apropriada do sinal amplificado, sendo constituído por dois componentes: o monitor, que converte o sinal amplificado de tal forma que a curva pressórica possa ser visualizada em tempo real, e o registrador, que possibilita documentar as informações de forma gráfica (jato de tinta, papel fotográfico ou termossensível ou sistemas digitalizados com feixes de raio laser).

Quando se registra uma curva de pressão sanguínea, o transdutor efetua a conversão da energia mecânica (sinal de entrada) em energia elétrica (sinal de saída). Os transdutores (manômetros eletromecânicos) acarretam distorções na onda portadora (sinal de entrada do amplificador), que precisa ser modulada, retificada e filtrada para gerar a curva de pressão que se observa no monitor e nos registros. Os sistemas manométricos, ao receberem o sinal de entrada, geram a cada instante um valor de sinal de saída, de tal forma que a morfologia resultante da curva de pressão depende, em grande parte, das características físicas de seus componentes (transdutor, amplificador e registrador). Assim, para o desempenho desejado de um sistema manométrico, diversas condições devem ser satisfeitas, entre as quais se destacam ${ }^{2,3,11}$ :

1. Estabilidade: a capacidade de se manter estável, sem variações da linha de base, quando colocado no padrão zero ou quando uma pressão constante é aplicada por tempo prolongado. Quanto menor a faixa de pressão utilizada, maior deve ser a estabilidade. Uma

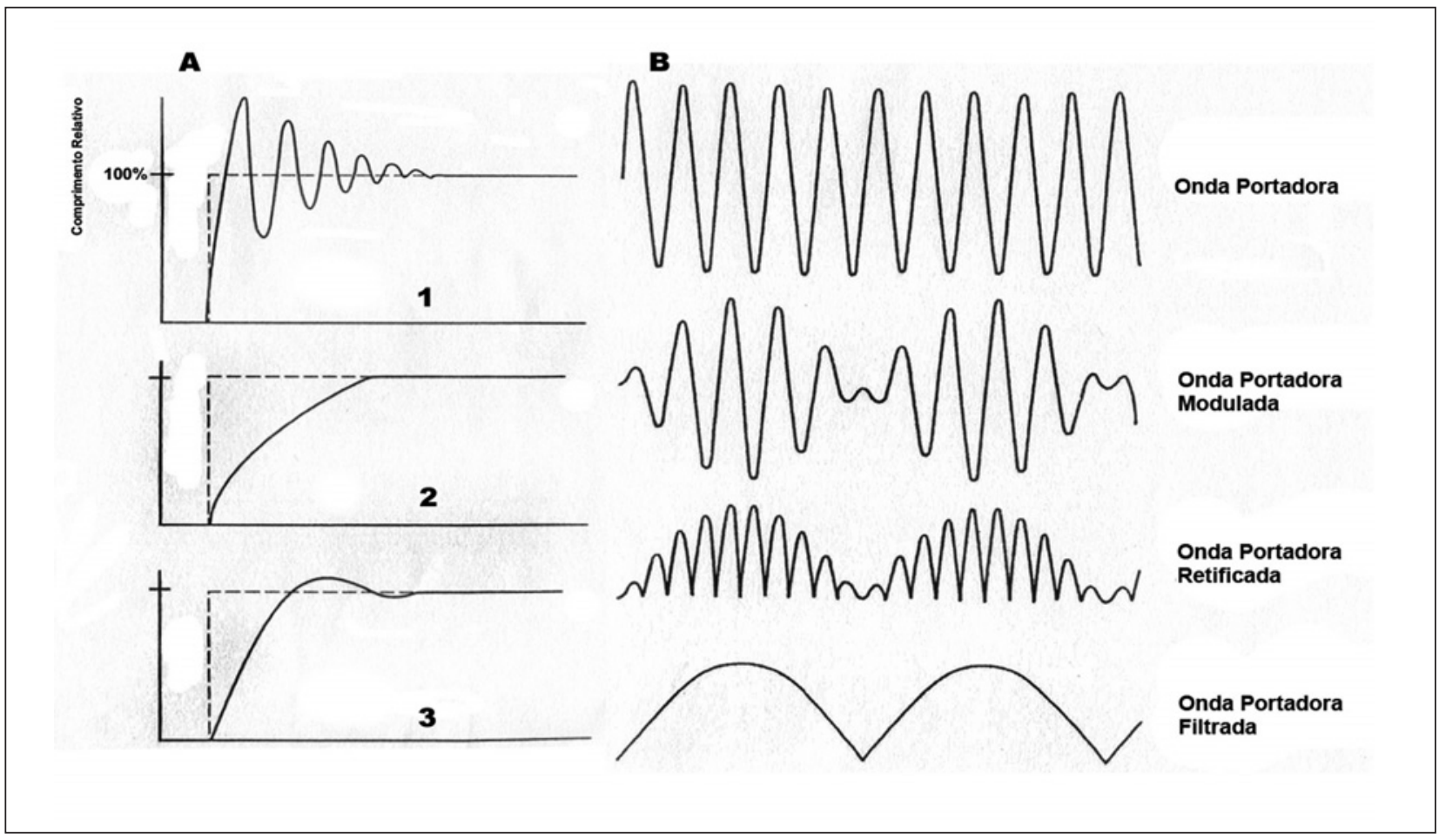

Figura 2 - A- Variação relativa da resposta de um sistema manométrico de acordo com o grau de amortecimento: 1. grau de amortecimento insuficiente; 2. grau de amortecimento excessivo; 3. grau de amortecimento adequado. B- Características dos amplificadores de pressão. A onda de pressão sinusoidal, inicialmente modulada, após ser retificada e filtrada, constitui a curva de pressão que se observa no monitor e é registrada. 
flutuação de $1 \mathrm{mmHg}$ da linha de base representa apenas $1 \%$, quando uma pressão de $100 \mathrm{mmHg}$ está sendo registrada; porém, a mesma variação equivale a $10 \%$ se a pressão registrada for de $10 \mathrm{mmHg}$.

2. Sensitividade: a capacidade de detectar pequenas variações do sinal de entrada e expressá-las em forma de respostas (sinais de saída) proporcionais às flutuações da variável que está sendo medida. Os instrumentos devem ser suficientemente sensíveis, para gerar sinais de saída adequados mesmo diante de pequenas variações dos sinais de entrada, e satisfatoriamente resistentes, para não serem danificados quando os sinais de entrada e/ou saída ultrapassarem a faixa de registro.

3. Linearidade: a linearidade existe quando a relação entre os sinais de entrada e saída do sistema pode ser expressa por uma equação de primeiro grau (linear) dentro da faixa de flutuação fisiológica das variáveis. Essa propriedade permite o uso de um único padrão de calibração para obtenção de todos os valores de pressões registrados. Os transdutores, amplificadores e registradores atuais satisfazem adequadamente a maioria desses critérios. Qualquer que seja o sistema de registro, a curva de pressão deve ocupar, aproximadamente, quatro quintos da altura do papel e pelo menos uma derivação eletrocardiográfica deve ser registrada simultaneamente, para, quando necessário, ser utilizada como referência cronológica. As curvas de pressão dos ventrículos direito e esquerdo devem ser registradas com velocidades mais altas $(25 \mathrm{~mm} / \mathrm{s}$ no mínimo), para se identificar e calcular com maior precisão os valores da pressão diastólica tanto inicial como final. Nos átrios, artérias e veias, além das curvas fásicas, também deve ser obtido o valor da pressão média, por amortecimento eletrônico presente nos equipamentos. Para registro das pressões pulmonar encunhada, das cavidades direitas e do átrio esquerdo é recomendável utilizar sensibilidade mais alta (padrão $10 \mathrm{mmHg}$ ) e tempo de registro que permita incluir pelo menos dois ciclos respiratórios. Caso isso não seja possível, devem ser considerados os valores no final da expiração. As pressões das diversas cavidades devem ser obtidas a partir da média aritmética dos valores de dez ciclos cardíacos consecutivos. Batimentos cardíacos que antecedem ou sucedem extra-sístoles não devem ser incluídos nos cálculos.

4. Flexibilidade e elasticidade: os cateteres são elásticos e flexíveis para possibilitar as manipulações necessárias em vasos e cavidades cardíacas. Se eles se expandirem com a elevação da pressão em seu interior, as características das curvas pressóricas não serão conduzidas fielmente em seu interior, gerando curvas pressóricas com distorções grosseiras, que poderão comprometer os valores reais da pressão e a morfologia das curvas, introduzir artefatos, e induzir erros nos resultados de variáveis cujos cálculos incluam os valores da pressão. Isso é particularmente comum quando se realiza cateterização cardíaca à beira do leito. É prática comum, nessas condições, a utilização de longos extensores, equipos de soro e diversas torneiras de três vias, que, aliados às pequenas dimensões da luz dos cateteres de balão flutuantes, reduzem de modo drástico a freqüência natural de ressonância, gerando curvas pressóricas extremamente amortecidas, valores de pressão inferiores aos reais, e artefatos e alterações morfológicas grosseiras. A utilização desses valores para o cálculo de vários parâmetros de função ventricular e de condições hemodinâmicas do paciente pode acarretar graves erros, capazes de induzir conduta inapropriada. Assim, é extremamente importante a cuidadosa atenção no preenchimento adequado de todo o sistema com fluidos, evitando-se, ao máximo, a presença de bolhas ou microbolhas. Além disso, na escolha de extensões e torneiras utilizadas, deve-se buscar manter o sistema com o menor volume de líquido possível. Como regra geral, pode-se afirmar que quanto mais rígido, de maior lúmen e mais curtos forem os dispositivos que conectam o paciente ao transdutor de pressão, maior e mais fidedignas serão a freqüência natural de ressonância do sistema, as morfologias e os valores das pressões. A Figura 3 ilustra as variações grosseiras na morfologia das curvas e dos valores da pressão do ventrículo esquerdo, quando o sistema manométrico contém, em seu interior, bolha de ar (Figura 3A), soro fisiológico (Figura 3B), contraste (Figuras 3C e 3D), durante elevação acentuada da freqüência ventricular (Figura 3E) e após administração do vasodilatador mononitrato (Figuras 3F e 3G). Essas variações são decorrentes de alterações da freqüência natural de ressonância, dependentes de modificações das características dinâmicas do sistema, introduzidas pelos diferentes componentes que o preenchem.

5. Freqüência natural de ressonância: a coluna líquida que preenche o sistema manométrico é a parte integrante mais crítica na determinação da freqüência natural de ressonância e a que acarreta os maiores problemas. Antes de ser adicionada, os sistemas apresentam boa estabilidade e freqüência natural de ressonância linear de até 1.000 cps. Quando o cateter, as torneiras e os extensores são adicionados e preenchidos com soro, os efeitos da coluna de fluido, a flexibilidade e a elasticidade de suas paredes são os principais fatores determinantes da freqüência natural de ressonância ${ }^{2,3,11}$ (Figuras 4A, 4B, 4C e 4D).

A pressão arterial e o fluxo sanguíneo variam ciclicamente, de forma regular e com determinada freqüência, podendo ser considerados formas de ondas. Nos ventrículos e grandes vasos, essas ondas têm contornos irregulares, que pouco se assemelham a ondas sinusoidais. No entanto, matematicamente, pode ser demonstrado que, na realidade, as curvas pressóricas são formadas por ondas sinusoidais superpostas, com possibilidade de serem decompostas em uma freqüência fundamental e seus múltiplos (harmônicos) 2,11,12. Essa decomposição pode ser realizada como uma série de Fourier, isto é, elas podem ser representadas como a 


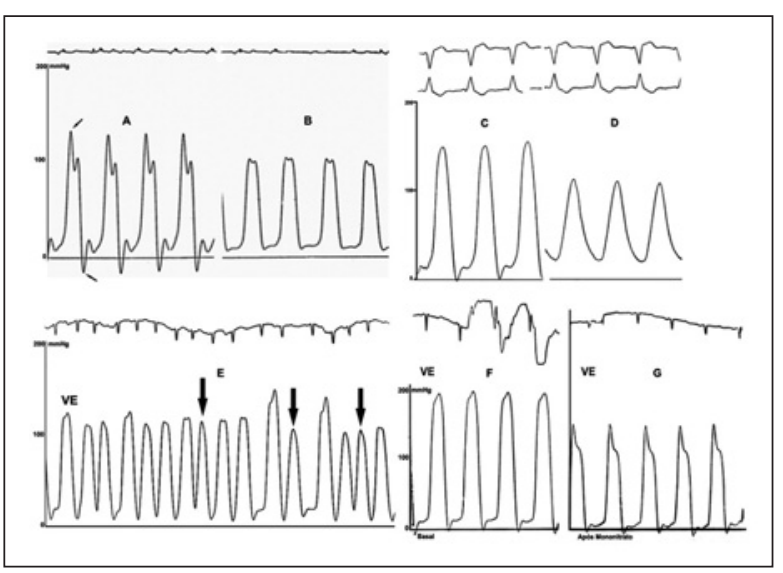

Figura 3 - Alterações morfológicas das curvas pressóricas do ventrículo esquerdo (VE) relacionadas às características do sistema manométrico. A- Presença de espículas (setas) no pico de pressões sistólica e diastólica pela presença de bolhas de ar. B- Mesmo paciente com o sistema corretamente preenchido com soro fisiológico. C- Curva de pressão com sistema corretamente preenchido. D- Mesmo paciente, quando o sistema contém contraste. E- Alterações da morfologia e dos valores relacionados a variações da freqüência cardíaca. Quando a freqüência cardíaca é elevada, as curvas pressóricas são amortecidas (setas); quando ela é menor, a morfologia é normal. FCurva de pressão do VE antes de mononitrato. G- Curva de pressão do VE após mononitrato. Observa-se modificação da morfologia decorrente da vasodilatação.

somatória de ondas sinusoidais de diferentes freqüências e amplitudes (Figura 5). As freqüências obtidas após a decomposição são a fundamental (freqüência cardíaca) e seus múltiplos (harmônicos). Exemplificando, se o valor da freqüência cardíaca é de 60 bpm, a freqüência fundamental é uma onda sinusoidal com freqüência de 1 cps. Quando a freqüência cardíaca for de 90 bpm ou 120 bpm, a freqüência fundamental será de 1,5 cps e 2 cps, respectivamente. Quando a freqüência fundamental for de $1 \mathrm{cps}$, o primeiro harmônico será de 1 cps, o segundo será de 2 cps, o terceiro será de 3 cps, e assim sucessivamente. Se a freqüência fundamental for de 2 cps, o primeiro harmônico será de 2 cps, o segundo harmônico será de 4 cps, o terceiro será de 6 cps, e assim sucessivamente. A contribuição relativa de cada harmônico para a morfologia resultante final das curvas pressóricas é progressivamente menor à medida que os harmônicos se sucedem. A freqüência fundamental e os dois primeiros harmônicos contribuem para $90 \%$ da morfologia final da curva de pressão na raiz da aorta, enquanto para se evidenciar adequadamente a incisura dicrótica, os seis primeiros harmônicos são importantes ${ }^{11,12}$. Por outro lado, sendo a curva de pressão do ventrículo esquerdo morfologicamente mais complexa, a obtenção de registros pressóricos com morfologia apropriada depende da contribuição de até o décimo harmônico. Por exemplo, para uma freqüência cardíaca de 120 bpm, os componentes mais importantes que contribuem para as características morfológicas das pressões aórtica e do ventrículo esquerdo são de 12 cps e 20 cps, respec- tivamente. Isso significa que os sistemas manométricos utilizados para a obtenção das pressões ventriculares precisam ter freqüência natural de ressonância mais elevada que aqueles utilizados para registros das pressões aórtica ou da artéria pulmonar.

Os sistemas manométricos devem ser capazes de registrar as ondas propagadas de forma tão rápida quanto os componentes de interesse que se pretende analisar, sem amplificação ou redução, característica conhecida como traçado livre de distorção de amplitude (resposta linear). Se um desvio de fase é produzido pelo sistema, ele deve afetar proporcionalmente todas as freqüências relativas, de tal modo que as relações temporais entre elas sejam preservadas e a morfologia da curva pressórica resultante seja uma reprodução da original, mesmo na vigência do desvio de fase ${ }^{2}$.

Para exames diagnósticos em humanos adultos, um sistema manométrico com freqüência natural de ressonância de 25 cps, geralmente, é adequado para obtenção da maioria das informações úteis derivadas das curvas de pressão, visto que para freqüências cardíacas de 120 bpm o décimo harmônico das curvas de pressão do ventrículo esquerdo equivale a 20 cps. O mesmo não pode ser afirmado quando o laboratório se destina a exames diagnósticos em crianças, avaliação hemodinâmica durante atividade física e estimulação cardíaca artificial, na vigência de taquiarritmias ventriculares, como, por exemplo, fibrilação atrial paroxística (Figura 3E), e nos laboratórios experimentais que utilizam pequenos animais. Nesses casos, os valores da freqüência cardíaca atingem cifras elevadas e os sistemas manométricos precisam ser especialmente projetados para possuir freqüência natural de ressonância suficientemente elevada para os objetivos a que se destinam. Na prática diária, a utilização de transdutores com câmara pequena (como, por exemplo, StathanP-50), o emprego de soro desborbulhado para o preenchimento do sistema manométrico, a não colocação ou redução de conectores ao mínimo, bem como cateteres curtos e mais calibrosos podem melhorar, substancialmente, as características dinâmicas do sistema manométrico. Quando disponível, o cateter manômetro é a solução mais apropriada para essas condições.

\section{MOVIMENTOS DA COLUNA DE FLUIDOS E RESSONÂNCIA}

As oscilações geradas pelas variações da pressão movimentam, de maneira cíclica, a coluna líquida no interior do cateter e a membrana do transdutor. Do lado oposto da membrana, existe um sistema eletrônico que converte os sinais mecânicos em elétricos, proporcionais à movimentação da membrana. Se a membrana for muito complacente, ela irá responder com grandes excursões, mesmo às pequenas variações pressóricas, e um volume relativamente grande de líquido irá ser movimentar, ciclicamente, no interior do sistema. Considerando-se que os cateteres são longos e possu- 
Bregagnollo EA, et al. Aspectos Metodológicos Relacionados aos Sistemas Manométricos Utilizados em Estudos Hemodinâmicos. Rev Bras Cardiol Invas 2007; 15(4): 421-431.

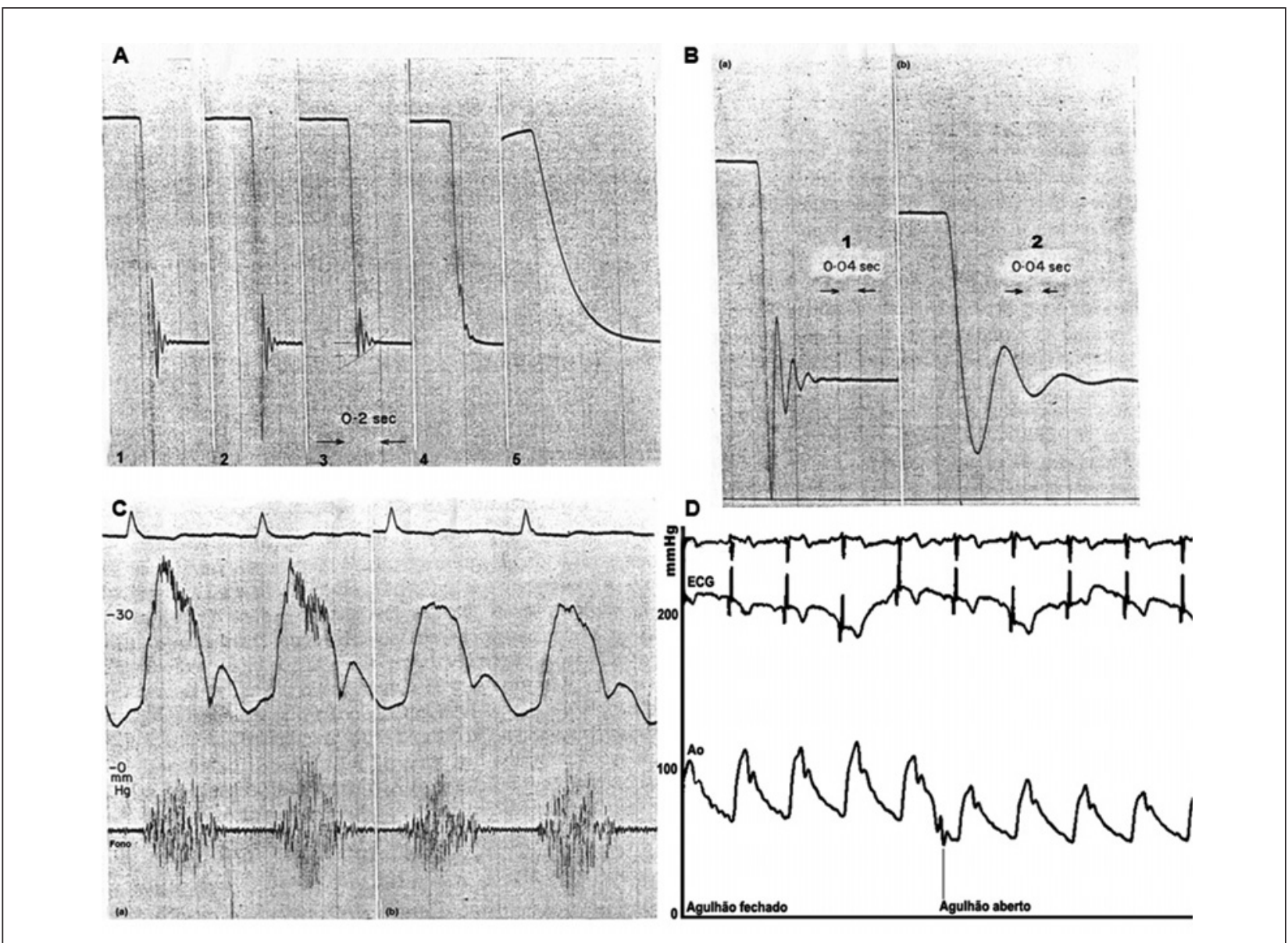

Figura 4 - A- Efeito do aumento progressivo do grau de amortecimento do sistema manométrico sobre uma onda quadrada registrada (cateter NIH-6F 125 cm): 1 e 2, baixo grau de amortecimento; 3, amortecimento adequado; 4 e 5, amortecimento excessivo. B- Antes e após a colocação da torneira de cinco vias. C- Pressão da artéria pulmonar de paciente (comunicação interatrial): 1 . fluxo turbulento - quando o grau de amortecimento é inadequado ocorre ressonância, que amplifica os valores pressóricos; 2. quando o amortecimento é correto, a curva pressórica não tem artefatos. D- Pressão aórtica durante angioplastia coronária com emprego do "agulhão" utilizado para manipulação do fio-guia. Na presença do "agulhão", há queda dos valores da pressão aórtica, sem modificações significativas da morfologia da curva.

em luz relativamente estreita, haverá retardo na transmissão da onda de pressão até o transdutor, que pode ser de até 20 mseg. Nessas condições, a membrana do transdutor poderá não ter retornado à posição inicial em tempo necessário para responder adequadamente à nova oscilação. Em outras palavras, haverá sensível redução da freqüência natural de ressonância, que resultará em alterações grosseiras nas características morfológicas das curvas de pressão registradas. Essa situação tende a se acentuar quando a freqüência cardíaca se eleva. Se a membrana do transdutor for muito rígida e sua área for pequena, a movimentação durante pequenas variações de pressão será mínima e o sinal elétrico de saída será pequeno. Quanto menor e mais rígida, maior será sua freqüência de resposta e maior a necessidade de amplificação do sinal de saída, facilitando a instabilidade e o aparecimento de ruídos que podem comprometer a morfologia das curvas pressóricas, principalmente quando não são elevadas, tais como as atriais e diastólica final do ventrículo esquerdo. Isso também pode ocorrer quando o aumento da rigidez do sistema acontece por enrijecimento da membrana do domus, em decorrência de múltiplas esterilizações. Nessa condição, além das alterações da morfologia das curvas de pressão, é comum se observar instabilidade da linha de base. Assim, o desempenho global de um sistema manométrico está na dependência das características do conjunto transdutorcateter-fluido que preenche o sistema.

Na prática, admite-se que sistemas manométricos de alta qualidade devem possuir freqüência natural de ressonância de 100 cps. Essa freqüência somente é obtida se cuidados extremos forem tomados durante o preenchimento do sistema com fluidos ${ }^{2,11,13}$, entre os quais cabe destacar: 1. o cateter deve ser conectado diretamente ao transdutor; 2. o fluido usado para encher o sistema precisa estar livre de bolhas ou microbolhas de ar e à mesma temperatura da sala de exame (a manei- 


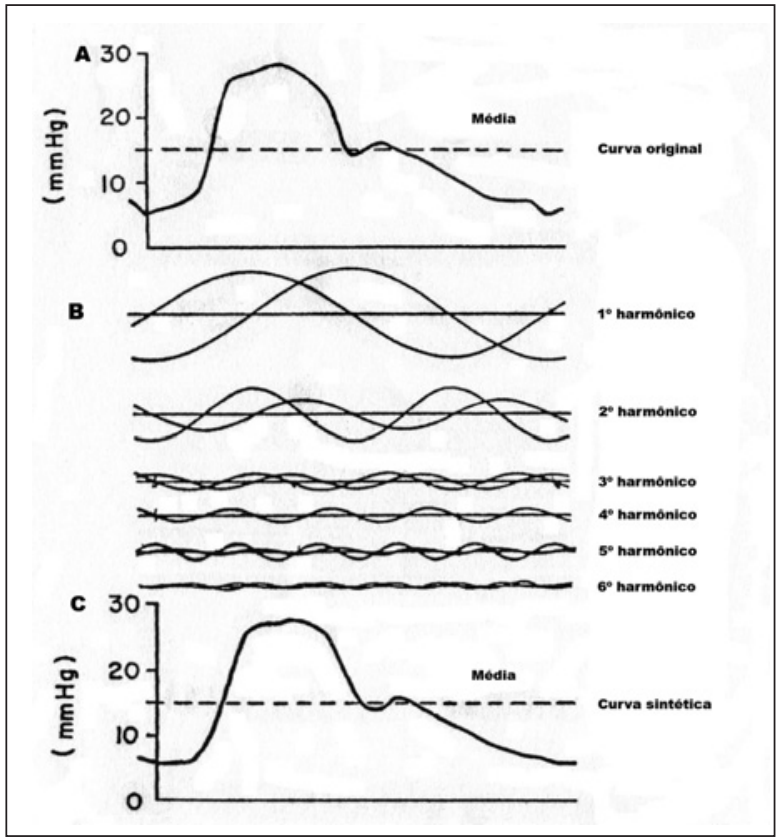

Figura 5 - Análise de Fourier. A- A curva superior é o traçado da pressão da artéria pulmonar e representa a somatória dos diversos componentes sinusoidais (harmônicos) que compõem a curva original. B- Decomposição da curva original em seus diversos harmônicos. CComposição eletrônica sintética da curva pressórica a partir dos harmônicos. Notar que a curva sintética é semelhante à original.

ra mais prática de se retirar o ar dissolvido no soro que irá preencher o sistema é aquecê-lo previamente, durante quatro a seis horas, e aguardar o esfriamento espontâneo, sem agitar o frasco); 3. preencher todo o sistema de modo lento e exclusivamente pela força da gravidade, para evitar que microbolhas de ar se prendam à parede do cateter ou domus; e 4. promover a passagem de $\mathrm{CO}_{2}$, durante cinco minutos, no interior do cateter e domus, previamente ao preenchimento. No entanto, a adesão a todos esses cuidados é inviável na prática diária para exames com objetivos puramente diagnósticos, principalmente em serviços com grande volume. Por outro lado, quando se utiliza a análise da morfologia e os valores pressóricos em investigações científicas, todos os cuidados citados devem ser rigorosamente observados, principalmente se a pressão ventricular esquerda é utilizada para obtenção da primeira derivada temporal $(\mathrm{dP} / \mathrm{dt})$ ou a ESF do ventrículo esquerdo como índices de contratilidade e/ou relaxamento miocárdico. Nessas condições, pequenos artefatos na curva de pressão do ventrículo esquerdo promovem enormes modificações dos valores desses índices ${ }^{14-17}$. Assim, quando se pretende avaliar o comportamento de variáveis como a $\mathrm{dP} / \mathrm{dt}$ ou a ESF, deve-se dar preferência ao uso do cateter manômetro.

Quando os componentes de freqüências mais elevadas (harmônicos) que compõem a curva de pressão analisada forem menores que a freqüência natural de ressonância, os traçados obtidos não apresentarão dis- torções significativas da morfologia e dos valores reais das pressões; quando superiores, as curvas apresentarão decréscimo das velocidades de ascensão e redução (amortecimento); e quando iguais ou muito próximos da freqüência natural de ressonância do sistema manométrico, os valores pressóricos podem ser amplificados $^{8,11,12}$ (Figuras 4B e 4C). Pelo exposto, poderse-ia concluir que o ideal seriam os sistemas manométricos que apresentassem o menor grau de amortecimento possível. No entanto, para a obtenção de traçados adequados, na prática diária, os sistemas manométricos precisam apresentar algum grau de amortecimento, por várias razões: 1. na presença de sopros, o sistema pode apresentar ressonância, pela alta freqüência dos mesmos (Figura 4C); 2. a conexão do cateter diretamente ao transdutor acarreta uma série de inconvenientes do ponto de vista tanto técnico como prático, pela necessidade de extensores e conectores, que sempre alteram as características dinâmicas do sistema (Figura 4B); 3. a movimentação da ponta do cateter no interior do sistema circulatório pode introduzir artefatos nos traçados; e 4. os métodos de lavagem do sistema durante os procedimentos não são perfeitos.

\section{GRAU DE AMORTECIMENTO DO SISTEMA}

Quando a freqüência natural de ressonância de um sistema manométrico for igual ou muito próximo a um componente importante da onda de pressão, o sistema pode ressoar e acarretar graves distorções na morfologia final da curva e nos valores das pressões registradas $1,2,11,13$. Isso pode ser prevenido "amortecendo-se" o sistema, por meio da introdução aleatória de constrições de graus variáveis próximas à entrada do transdutor, com o intuito de evitar a movimentação rápida da coluna líquida e, assim, reduzir as oscilações com freqüências próximas à freqüência natural de ressonância. Outro modo é a colocação de filtros eletrônicos, passa-baixos ou passa-altos, existentes como recursos em polígrafos mais sofisticados. Esses filtros possibilitam trabalhar em faixa de freqüência determinada. Por exemplo, a colocação de filtros que limitam fenômenos vibratórios com freqüências acima de $250 \mathrm{~Hz}$ (filtros passa-baixos) e abaixo de $20 \mathrm{~Hz}$ (filtros passaaltos) excluem, respectivamente, fenômenos vibratórios com freqüências acima e abaixo das estipuladas. Para um dado sistema manométrico, quando o grau de amortecimento é gradualmente aumentado, os artefatos resultantes de ressonância irão progressivamente reduzir em tamanho até desaparecer. Amortecimentos adicionais podem diminuir a freqüência natural de ressonância de tal forma que, embora visualmente não afete de modo significativo os traçados, podem não conter alguns componentes de alta freqüência (incisura dicrótica). Isso afeta de modo expressivo os valores da $\mathrm{dP} / \mathrm{dt}$. Quando o amortecimento é excessivo, a velocidade de ascensão e redução das ondas pressóricas também será lentificada. Isso pode ser facilmente observado pela presença de contraste no interior do cate- 
ter (Figuras 3C e 3D) ou na presença do "agulhão" usado para auxiliar a manipulação de fio-guia durante angioplastia coronária (Figura 4D).

Teórica e experimentalmente, está demonstrado que um sistema manométrico que registra ondas quadradas com somente $5 \%$ a $7 \%$ de excesso (overshoot) possibilita registros pressóricos de boa qualidade e com desvios de fase proporcionais dos diversos harmônicos que compõem as curvas de pressão. Esse grau de amortecimento é denominado "amortecimento ótimo"12-14.

\section{MÉTODOS DE DETERMINAÇÃO DA FREQÜÊNCIA NATURAL DE RESSONÂNCIA E DO GRAU DE AMORTECIMENTO}

1. Método da oscilação forçada: utiliza um gerador de ondas sinusoidais acoplado ao sistema manométrico ${ }^{1,11,18}$. Esse método submete o sistema a ondas sinusoidais com amplitude uniforme e freqüências progressivamente mais elevadas, que são registradas em papel, com velocidade de transporte de $200 \mathrm{~mm} /$ s ou mais. Quando se utiliza esse método, a determinação da freqüência natural de ressonância é simples e facilmente visualizada (Figura 6A). Nessa situação, a redução da freqüência natural de ressonância pela presença de bolhas de ar no líquido que preenche o sistema é facilmente demonstrada (Figuras 6A e 6B).

2. Método da oscilação livre: por esse método, uma variação súbita da pressão é aplicada ao sistema manométrico utilizando-se seringa de vidro cujo bico metálico foi retirado, possibilitando a colocação da ponta do cateter no interior do cilindro, sendo este firmemente fixado em suporte rígido. A seguir, o orifício pelo qual a ponta do cateter penetra é ocluído com anel de borracha. Nos extremos do êmbolo, são feitos orifícios e o sistema cateter-transdutor é cuidadosamente preenchido com soro fisiológico ou glicosado. O êmbolo é introduzido no cilindro da seringa, o orifício do topo

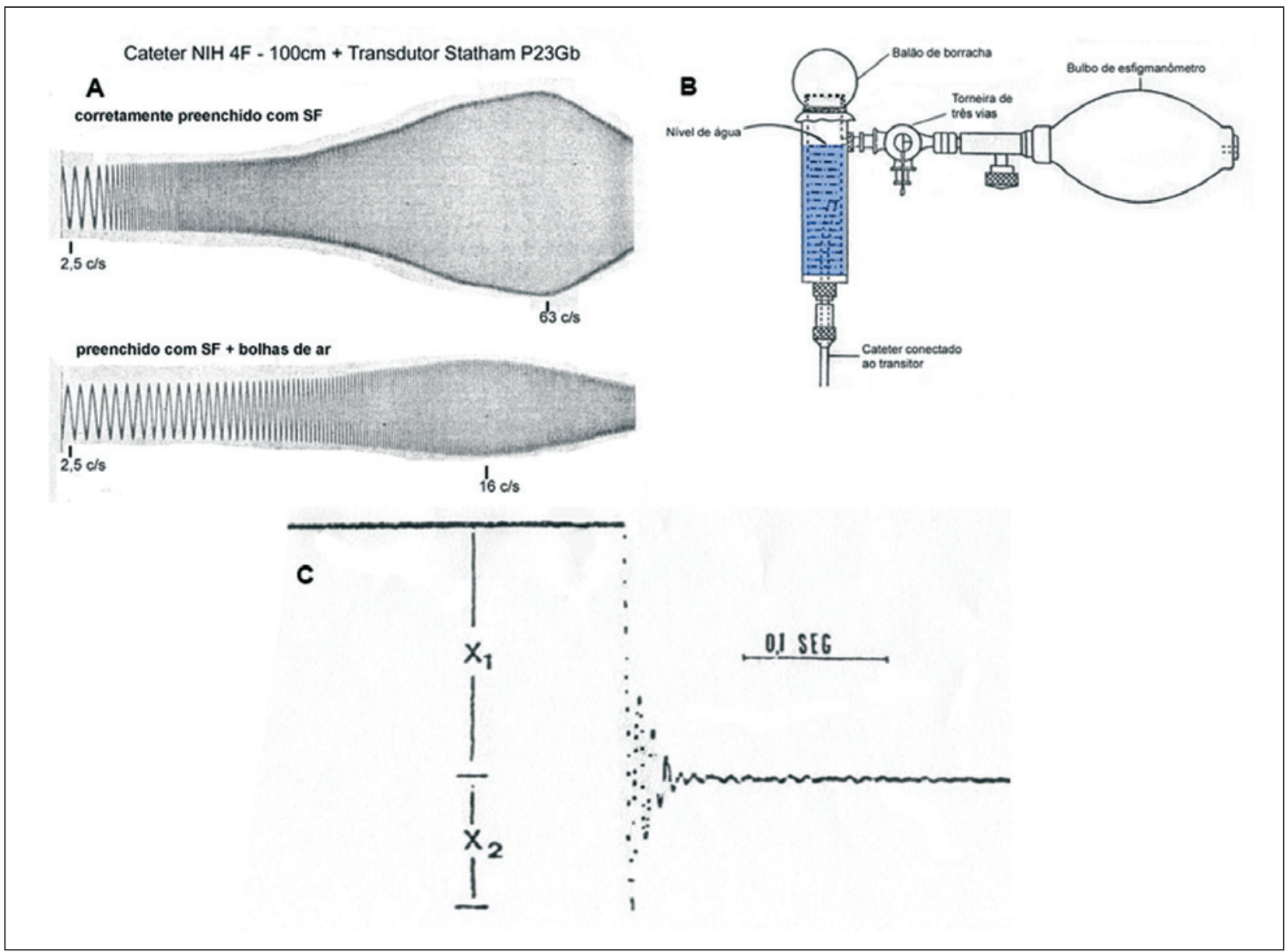

Figura 6 - A- Amplitude de resposta de um sistema de pressão (cateter NIH 4F) após aplicação de uma onda sinusoidal, elevando-se progressivamente a freqüência. Quando o sistema é corretamente preenchido, a frequêência natural de ressonância (F) é de 63 cps; quando pequena quantidade de ar é introduzida no mesmo, a $\mathrm{F}$ cai para $16 \mathrm{cps}$. B- Dispositivo usado em laboratório experimental para avaliar as características dinâmicas do sistema manométrico (método do "estouro do balão"). C- Resposta oscilatória do sistema. A velocidade de transporte do papel é de $250 \mathrm{~mm} / \mathrm{s}$. Nesse sistema, $\mathrm{F}=125 \mathrm{cps}$, grau de amortecimento $(\mathrm{h})=0,18$ e overshoot $=8 \%$, o que possibilita a obtenção de registros pressóricos sem distorções significativas, com valores de freqüência cardíaca de até 600 batimentos por segundo. 
Bregagnollo EA, et al. Aspectos Metodológicos Relacionados aos Sistemas Manométricos Utilizados em Estudos Hemodinâmicos. Rev Bras Cardiol Invas 2007; 15(4): 421-431.

é ocluído com o dedo e, a seguir, lentamente retirado, gerando pressão subatmosférica no sistema. No momento em que o êmbolo é retirado do cilindro, a pressão retorna subitamente aos valores atmosféricos, gerando oscilações cuja amplitude e freqüência possibilitam calcular a freqüência natural de ressonância e o grau de amortecimento do sistema manométrico ${ }^{12}$.

3. Método do estouro do balão: uma seringa firmemente fixada é parcialmente preenchida com solução fisiológica ou glicosada (5\%), de modo a encobrir a extremidade livre do cateter. Com o conjunto montado nas mesmas condições de uso rotineiro, insufla-se um balão de borracha com ar, gerando hipertensão no sistema. Com o auxílio de uma chama, estoura-se o balão, provocando queda súbita da pressão até valores atmosféricos ${ }^{2}$. Nota-se que a queda de pressão, que deveria atingir apenas a linha de referência zero, ultrapassa essa linha e é seguida de movimento oscilatório resultante da ressonância da coluna líquida no interior do sistema manométrico. As características da amplitude e a freqüência desse movimento oscilatório permitem calcular a freqüência natural de ressonância e o grau de amortecimento do sistema manométrico que está sendo utilizado (Figura 6C).

\section{CÁLCULO DA FREQÜÊNCIA NATURAL DE RESSONÂNCIA E DO GRAU DE AMORTECIMENTO DO SISTEMA MANOMÉTRICO}

A Figura 7 ilustra a resposta oscilatória de um sistema manométrico após aplicação de uma variação súbita de pressão sob a forma de onda quadrada, utilizando um dos métodos descritos anteriormente. Para a determinação das características dinâmicas do sistema manométrico, são utilizadas as fórmulas apresentadas na Figura 7, onde: $\mathrm{h}=$ grau de amortecimento do sistema, $F=$ freqüência natural de ressonância, $p=$ período das oscilações, $F d=1 / p$, e pi $=3,142$. A Figura 8 demonstra um exemplo de cálculo do grau de amortecimento e da freqüência natural de ressonância de um sistema manométrico.

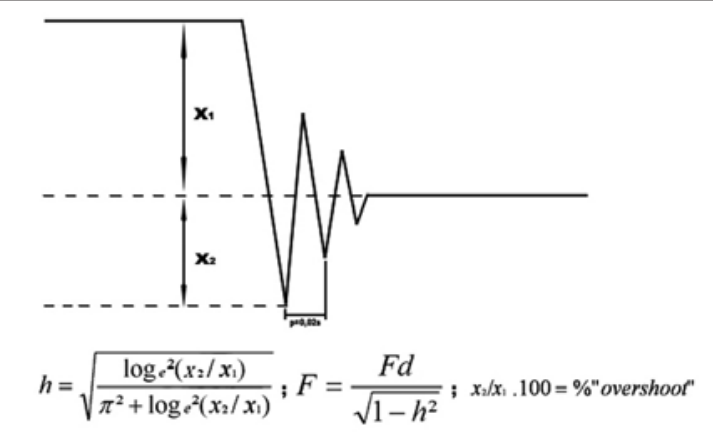

Figura 7 - Representação esquemática da resposta oscilatória de um sistema manométrico obtida após aplicação de uma onda pressórica quadrada. Para cálculo das características dinâmicas de um sistema manométrico, vide exemplo no texto. $F=$ freqüência natural de ressonância; $p=$ período das oscilações $(s) ; F d=1 / p ; p i=3,142$.

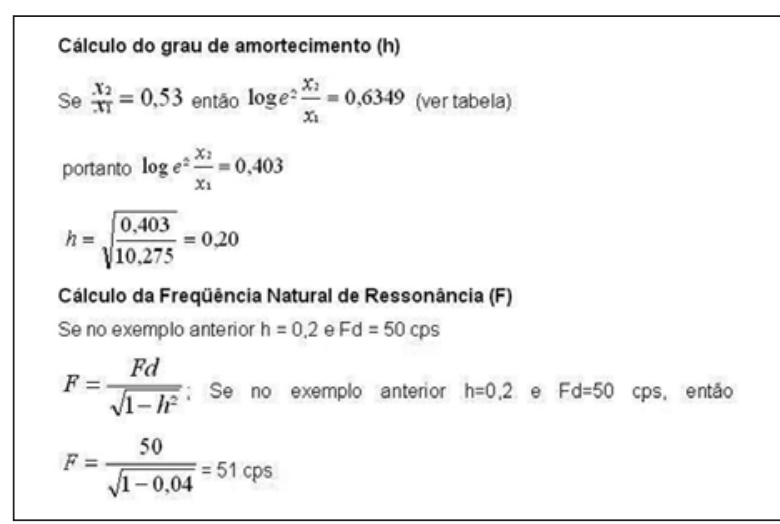

Figura 8 - Cálculo do grau de amortecimento (h) e da freqüência natural de ressonância (F) de um sistema manométrico.

\section{REFERÊNCIAS BIBLIOGRÁFICAS}

1. Bertrand ME, Widimsky J. Pressure. Eur Heart J. 1985;6(supl C):5-9.

2. Yang SY, Bentivoglio MS, Maranhão V, Goldberg H. Basic measurement and calculations. In: Yang SY, Bentivoglio MS, Maranhão $\mathrm{V}$, Goldberg $\mathrm{H}$, eds. From cardiac catheterization data to hemodynamic parameters. $2^{\text {nd }}$ ed. Philadelphia: Davis;1978. p.1-54.

3. Moreno MG, Matinez-Rios MA. Registros de pressiones, flujos y resistências em el aparato cardiovascular. In: MatinezRios MA, ed. Cateterismo cardíaco: diagnóstico e tratamento das cardiopatias. 2a ed. México:Trillas;1997. p.53-69.

4. Bregagnollo EA, Matsubara BB, Padovani C, Tucci PJ. Influência da elevação transitória e da elevação sustentada da pressão arterial sobre a primeira derivada temporal da pressão ventricular esquerda. Arq Bras Cardiol. 1992;58(6):437-43.

5. Bregagnollo EA, Okoshi K, Matsubara BB, Tucci PJ. A elastância sistólica final do ventrículo esquerdo determinada durante elevações transitórias e sustentadas da pressão arterial. Arq Bras Cardiol. 2000;75(1):19-32.

6. Matsubara BB, Bregagnollo EA, Ribeiro MA, Tucci PJ. Relações pressão/diâmetro e esforço/diâmetro do ventrículo esquerdo em seres humanos. Padronização e análise crítica do método. Arq Bras Cardiol. 1990;54(6):373-81.

7. Matsubara BB, Bregagnollo EA, Ribeiro MA, Tucci PJ. Left ventricular maximal systolic elastance calculated by a combination of M-mode echocardiography and standard manometry. Braz J Med Biol Res. 1991;24(4):383-94.

8. Dear HD, Spear AF. Accurate method for measuring $d P / d t$ with cardiac catheters and external transducers. J Appl Physiol. 1971;30(6):897-9.

9. Chandraratna PA. Determination of zero reference level for left atrial pressure by echocardiography. Am Heart J. 1975;89 (2):159-62.

10. Shapiro GG, Krovetz LJ. Damped and undamped frequency responses of underdamped catheter manometer systems. Am Heart J. 1970;80(2):227-36.

11. Mendel D. Measurement of pressure. In: A practice of cardiac catheterization. Oxford:Blackwell Scientific Publications;1968. p.207-47.

12. Fry DL. Physiologic recording by modern instruments with particular reference to pressure recording. Physiol Rev. 1960; 40:753-88.

13. Grossman W. Pressure measurement. In: Grossman W, Baim DS, eds. Cardiac catheterization angiography and intervention. $7^{\text {th }}$ ed. Philadelphia:Williams \& Wilkins;2006. p.133-47. 
Bregagnollo EA, et al. Aspectos Metodológicos Relacionados aos Sistemas Manométricos Utilizados em Estudos Hemodinâmicos. Rev Bras Cardiol Invas 2007; 15(4): 421-431.

14. Yanof HM, Rosen AL, McDonald NM, McDonald DA. A critical study of the response of manometers to forced oscillations. Phys Med Biol. 1963;66:407-22.

15. Hansen AT. Pressure measurement in the human organism. Acta Physiol Scand. 1949;19(suppl 68):87-92.

16. Mason DT. Usefulness and limitations of the rate of rise of intraventricular pressure $(\mathrm{dP} / \mathrm{dt})$ in evaluation of myocardial contractility in man. Am J Cardiol. 1969;23(4):516-27.
17. Spann JF, McCusteker DR, Zelis R, Mason DT. Determination of maximum contractile element shorting in man utilizing standard catheter techniques: application of a new external microdisplacement transducer allowing high fidelity intracardiac pressure recordings. Circulation. 1969;40(suppl 3):90-8.

18. Sonnenblick EH, Parmley WW, Urschell CW, Brutsaert DL. Ventricular functions: evaluation of myocardial contractility in health and disease. Prog Cardiovasc Dis. 1970;123:449-66. 